\title{
INTELIGENCIA ARTIFICIAL PARA LA ADMINISTRACIÓN DE LOS REGLAMENTOS AERONÁUTICOS DE COLOMBIA (RAC)
}

\author{
ARTIFICIAL INTELLIGENCE FOR \\ THE ADMINISTRATION OF THE AERONAUTICAL \\ REGULATIONS OF COLOMBIA (RAC)
}

Recibido: 8 de agosto de 2020

Evaluado: 7 de septiembre de 2020

Aprobado: 14 de noviembre de 2020

\section{Paulino Rodríguez-Palomino **}

Aeronáutica Civil de Colombia

Orcid: https://orcid.org/0000-0002-9496-6708

Cómo citar este artículo: Rodríguez-Palomino, P. (2021). INTELIGENCIA ARTIFICIAL PARA LA ADMINISTRACIÓN DE LOS REGLAMENTOS AERONÁUTICOS DE COLOMBIA (RAC). Revista Estrategia Organizacional, 10(1), doi: https://doi. org/10.22490/25392786.4551

* $\quad$ Artículo de investigación producto del proyecto titulado: Solución informática con inteligencia artificial para resolver consultas normativas en el ámbito de los Reglamentos Aeronáuticos de Colombia (RAC).

** Administrador de Empresas. Magister en Administración de Organizaciones. Controlador de Tránsito AéreoSupervisor, Unidad Administrativa Especial de Aeronáutica Civil-Colombia. Correo electrónico: paulino.rodriguez@ aerocivil.gov.co 


\title{
RESUMEN
}

Introducción: Lo que se pretende con el presente artículo, es evidenciar los resultados hallados en un proyecto de consultoría a la Aerocivil. Metodología: se realizó un estudio de caso de negocio para determinar si es posible encontrar una solución informática con Inteligencia Artificial que permita obtener respuestas en tiempo real sobre consultas que se tengan acerca de la aplicación a casos específicos de las normas contenidas en los Reglamentos Aeronáuticos de Colombia (RAC). Resultados: Es por esto que el proyecto realizó una consultoría organizacional a la Aerocivil en dónde se identificó de forma adecuada el problema técnico en el que está inmersa, se desarrolló por medio de un caso de negocio la posible solución al problema técnico y los aspectos administrativos asociados, que determinaron su viabilidad y mostraron las posibles aplicaciones en la gestión de diferentes organizaciones.

Palabras clave: inteligencia artificial, minería de texto, procesamiento de lenguaje natural, derecho aéreo, transporte aéreo, administración.

\begin{abstract}
Introduction: The aim of this article is to show the results found in a consulting project for Aerocivil. Methodology: a business case study was carried out to determine if it is possible to find a computer solution with Artificial Intelligence that allows obtaining answers in real time on queries that are had about the application to specific cases of the rules contained in the Aeronautical Regulations of Colombia (RAC). Results: This is why the project carried out an organizational consultancy to Aerocivil where the technical problem in which it is immersed was properly identified, the possible solution to the technical problem and administrative aspects were developed through a business case associates, who determined its viability and showed the possible applications in the management of different organizations.
\end{abstract}

Keywords: artificial intelligence, text mining, natural language processing, air law, air transport, administration. 


\section{INTRODUCCIÓN}

El transporte aéreo colombiano está enmarcado en unas normas específicas para ser cumplidas por toda la comunidad aeronáutica, estas normas están contenidas en los Reglamentos Aeronáuticos de Colombia (RAC) que son estructurados por la Unidad Administrativa de Aeronáutica Civil de Colombia (Aerocivil). A pesar que las actividades propias de la aviación son complejas, también requieren ser ágiles con el fin de mantenerse competitivas y al mismo tiempo deben ser altamente seguras; la comunidad aeronáutica no puede darse el lujo de omitir el acatamiento a una norma que pueda violar el mínimo estándar de seguridad. Este compendio de normas está compuesto por 52 partes, con un total de 5831 páginas (a la fecha) y tienen la característica de ser actualizadas muy frecuentemente debido a los avances realizados en todas las áreas de la industria. Esto hace que el personal que trabaja en la aviación deba permanecer en actualizaciones continuas y dependen de su memoria para recordar las normas que los rigen. En ciertas situaciones donde se deben tomar decisiones de forma ágil y que existe la preocupación de mantener el estándar de seguridad, muchas veces las decisiones se dilatan con el fin de ir a la fuente de consulta del RAC para asegurarse que se cumple con la legislación, esta acción hace que se pierda eficacia en los procesos y puede generar un efecto dominó que repercute en costos y algunas veces en seguridad (Lo que resulta paradójico porque es lo que se quería proteger desde el principio).

Esto hace necesario que la Aerocivil, como encargada de establecer políticas que permitan garantizar el correcto funcionamiento de la aviación civil bajo principios de seguridad, oportunidad y eficiencia, se plantee la posibilidad de implementar un sistema informático con un componente de Inteligencia Artificial que esté capacitado para entender una consulta en lenguaje natural, realizar análisis de texto en el RAC y dé como entregable una respuesta en tiempo real y alineada con la normatividad. 


\section{METODOLOGÍA}

Para la realización del proyecto se completaron varias fases (Rodríguez, 2020): La primera fue la identificación del problema técnico, donde se describió el problema por medio de herramientas como el árbol de problemas, para luego identificar a los interesados con herramientas como la Matriz de poder-interés y la Matriz influencia-previsibilidad y se encontró una solución informática adecuada por medio de una revisión de fuentes documentales, se identificaron las posibles modalidades de solución teniendo en cuenta el impacto, la efectividad y las restricciones que debe enfrentar. Seguidamente, se hizo la presentación del caso de negocio siguiendo los lineamientos del Business Case Institute Scientific Management. Posteriormente, se dio solución al problema técnico, realizando un análisis de escenarios, análisis de causas y efectos y se verificó que la solución se alineara con los objetivos estratégicos de la Aerocivil. Por último, se revisaron todos los aspectos administrativos involucrados.

\section{RESULTADOS}

La organización sobre la cual se realizó el proyecto es la Unidad Administrativa Especial de Aeronáutica Civil (Aerocivil), la cual es una entidad del estado colombiano, que tiene como finalidad vigilar y controlar el espacio aéreo de Colombia y establecer políticas que permitan garantizar el correcto funcionamiento de la aviación civil bajo principios de seguridad, oportunidad y eficiencia. Según el Código de Comercio (1971), la Aerocivil es la "Autoridad Aeronáutica" en Colombia; y de acuerdo al Decreto 823 (2017, art. 1), tendrá doble función: como autoridad normativa y como prestadora de servicios (aeronáuticos y aeroportuarios).

\section{IDENTIFICACIÓN DEL PROBLEMA TÉCNICO}

\section{ÁRBOL DE PROBLEMAS}

Según la UNESCO (s.f.), un árbol de problemas es "una técnica que se emplea para identificar una situación negativa (problema central), la cual se intenta solucionar analizando relaciones de tipo causa-efecto", por lo que se decidió utilizar esta técnica para la definición del problema técnico. 


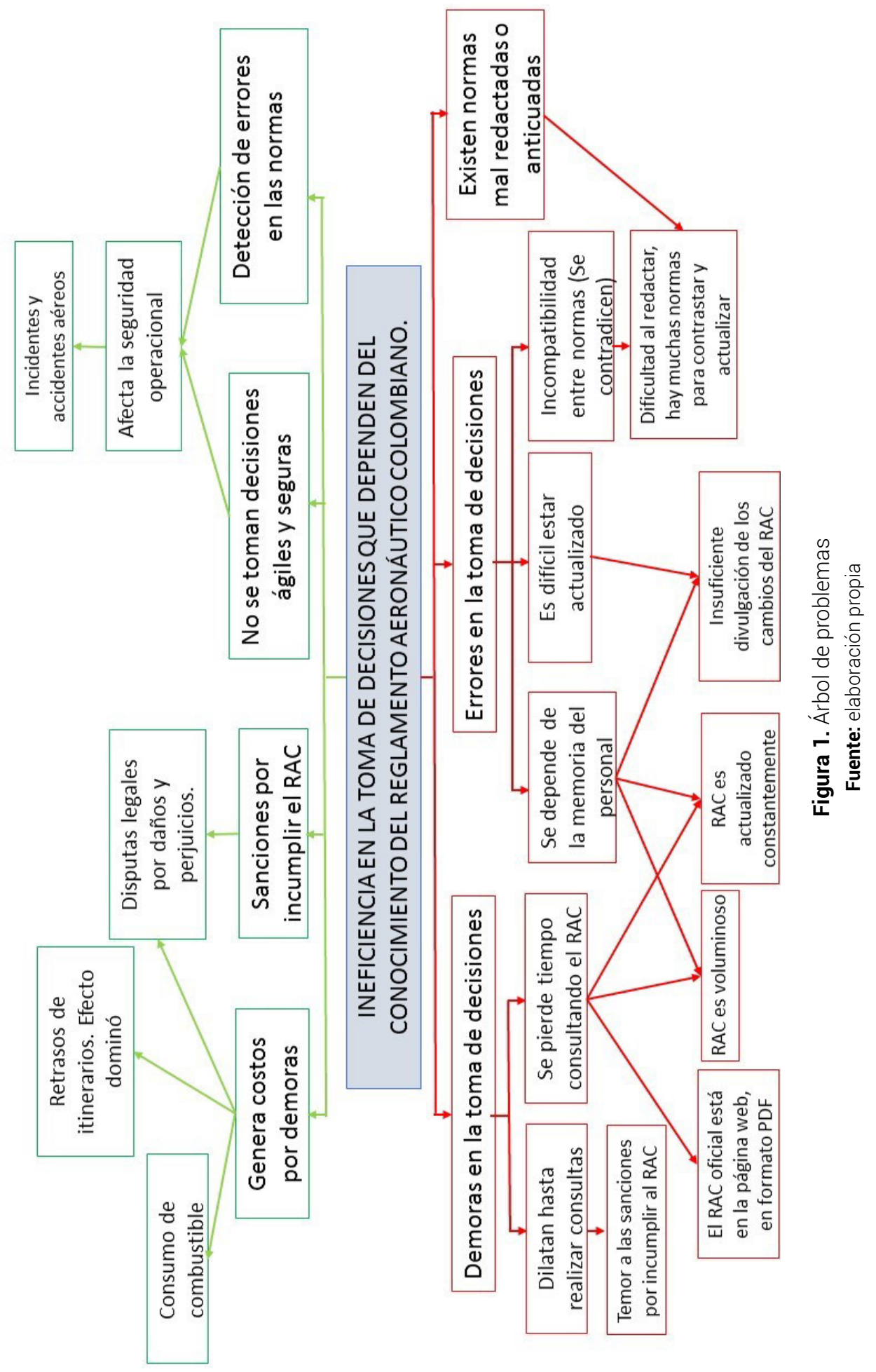




\section{ANTECEDENTES DEL PROBLEMA}

La práctica de cualquier actividad humana involucra el conocimiento apropiado de la regulación exigida por las autoridades, y esto está reforzado por un principio fundamental del derecho que es mencionado por Texo (2017): "el error iuris nocet, que se traduce en que la ignorancia de la ley no excusa", esto implica que los individuos deben conocer TODA la normatividad que involucra las labores en que están involucrados so pena de caer en ilegalidades. Si observamos la realidad, notamos que pretender conocer toda la normativa puede ser utópico y depende de la capacidad de acceso a la información y del afán personal o institucional por mantenerse actualizado. Ante este asunto, Texo (2017) indica que los estados deberían buscar otras formas de distribuir el saber jurídico y que se debe acercar el contenido de las normas a las personas por los medios que sean necesarios.

Este dilema cae en el marco antropológico de la cognición y la comunicación. Fabra y Núñez (2015) mencionan que suelen aceptarse cuatro grandes marcos generales y sucesivos: la oralidad, la escritura, la imprenta y las tecnologías de la información. Es en este marco de las tecnologías de la información en donde se puede automatizar de manera más efectiva el modo de capturar, compartir y comunicar la información relacionada con la normatividad aplicable a las labores en que estamos interesados. Para el uso específico en el manejo del derecho, dentro de las tecnologías de la información ha tomado gran relevancia el uso de la Inteligencia Artificial para ayudar a solucionar varios problemas:

... la organización de las grandes bases de datos, la clasificación y ordenación de textos jurídicos que varían con el tiempo (leyes, reglamentos, sentencias...), el interfaz con los distintos tipos de usuarios, el modelado de las operaciones realizadas por los agentes políticos (legística) y jurídicos (razonamiento judicial, razonamiento por casos, resolución de problemas), el modelado de determinados ámbitos del conocimiento jurídico (especialmente en derecho financiero y en cálculo de tasas e impuestos), el modelado de la argumentación razonable en función de normas o de precedentes. (Fabra y Núñez, 2015) 
También Fernández y Boulat (2015) han tratado el tema de sistemas que utilizan la Inteligencia Artificial en la rama del derecho y el modo en que esta ciencia afectará la labor de los juristas y de los ciudadanos en el futuro. Desde siempre ha existido el temor del desplazamiento del hombre por la labor de las máquinas, pero este temor queda descartado por el momento debido a las capacidades únicas de razonamiento que posee la raza humana; sin embargo, es indiscutible la ayuda que este tipo de tecnologías “...capaces de analizar a gran velocidad enormes volúmenes de documentación, para extraer de ellos pautas o mejores opciones de actuación ante supuestos concretos..." (Fernández y Boulat, 2015). Además, el enfoque con que se usa la tecnología ha cambiado de ser un reemplazo a ser un apoyo, una especie de colaboración hombre-máquina para la solución apropiada de problemas.

Hernández et al. (2019) intenta analizar los llamados Sistemas Expertos y su aplicación en la toma de decisiones jurídicas. Concluyó que las bases de datos jurídicas poseen una gran cantidad de información que un ser humano no puede tener presente en todo momento y es donde los Sistemas Expertos se convierten en una herramienta apropiada para ayudar en la toma de decisiones de carácter normativo.

\section{CONTEXTO DE DESARROLLO DEL PROBLEMA}

El transporte aéreo es un ecosistema que tiene muchos participantes, cada uno de ellos hace que el sistema funcione acorde con los altos estándares exigidos por la industria.

Con el objetivo que todos ellos cumplan a cabalidad con sus objetivos, deben actuar de acuerdo a un estricto cumplimiento de la norma, representado por los Reglamentos Aeronáuticos de Colombia (RAC). Según la Organización de Aviación Civil Internacional (OACl, 2005), la inmediatez de las decisiones que se tienen que tomar implica que el acceso a la información debe ser oportuna, pertinente, precisa, acreditada y con garantía de calidad. Es por esto que la Aerocivil, y basado en sus objetivos estratégicos, debería implementar un sistema que le permita a la comunidad aeronáutica solucionar de manera eficiente cualquier consulta en base a la norma. Adicionalmente, como ventaja, la Aerocivil es la encargada de redactar la normatividad, por lo que un sistema como el planteado ayudaría en el proceso de proyectar nuevas normas en cuanto a evitar incompatibilidades con el resto de documentación (lo que sucede a veces). 
Es importante tener en cuenta que el modo transporte aéreo es considerado un servicio público esencial (Artículo 68 de la Ley 336 de 1996), por lo que, cualquier perturbación o demora puede generar afectaciones graves en lo económico, social y ambiental; debido a esto, el transporte aéreo se convierte en un pilar fundamental para la conectividad del país tanto entre sus regiones como con el mundo.

\section{DESCRIPCIÓN DEL PROBLEMA}

El derecho aeronáutico abarca un conjunto limitado de normas. El artículo 68 de la Ley 336 de 1996, indica; “El modo de transporte aéreo, además de ser un servicio público esencial, continuará rigiéndose exclusivamente por las normas del Código de Comercio (libro quinto, capítulo preliminar y segunda parte), por el manual de reglamentos aeronáuticos que dicte la Unidad Administrativa Especial de Aeronáutica Civil, y por los tratados, convenios, acuerdos, prácticas internacionales debidamente adoptados o aplicadas por Colombia". Es decir, para el transporte aéreo existe una legislación definida y enmarcada, no tan extensa como las demás ramas del derecho. A pesar de no ser tan extensa, estamos hablando de una gran cantidad de literatura, en especial la de los llamados Reglamentos Aeronáuticos de Colombia (RAC), que consisten en un compendio de normas que busca abarcar toda la reglamentación técnica y administrativa que regulan aspectos propios de la aviación civil, en concordancia con otras normas nacionales e internacionales.

El problema de mantenerse actualizado en la ley con el fin de actuar de acuerdo a ésta, es un problema general que afecta a toda la población colombiana y a todas las ramas del derecho, como lo dijo Narváez (2018): “...cerca de 5.967 .000 normas expedidas en Colombia desde la creación del Congreso de la República, así como los miles de sentencias proferidas por jueces y magistrados de todo el país. No es posible exigir a ningún abogado, funcionario judicial o residente en Colombia que tenga pleno conocimiento de toda esta normativa y jurisprudencia, así lo exija la regla del Código Civil colombiano que establece que la ignorancia de la ley no sirve de excusa."

En las demás ramas del derecho y en la gran mayoría de las actuaciones, este impase es relativamente solucionable porque para tomar decisiones, la misma ley permite plazos de respuesta medidos en días, lo que da el espacio suficiente para indagar; pero en el área específica de la prestación de los servicios para la navegación aérea y aeroportuaria, donde los tiempos de respuesta y eficacia se mide en minutos (o por mucho, en horas), es muy importante para los participantes del ecosistema aeronáutico que exista la posibilidad de despejar dudas normativas en tiempo real. 
Actualmente, si alguna persona tiene una duda normativa debe dirigirse a la página web de la Aerocivil, navegar hasta la sección del RAC donde encontrará 52 archivos en PDF que conforman cada una de sus partes (Mediante Resolución 05036 del 18 de septiembre de 2009, la Aerocivil resuelve que: “...la versión oficial de los Reglamentos Aeronáuticos de Colombia (RAC) será aquella que la Unidad Administrativa Especial de Aeronáutica Civil publique en su página web. (www.aerocivil.gov.co)"), y posteriormente realizar la búsqueda de la respuesta adecuada en estos documentos, esto hace que se desperdicie tiempo valioso, el cual se traduce en aumento de costos, consumo de combustible, retraso de itinerarios, posibles cancelaciones de itinerarios posteriores, posibles disputas legales por daños y perjuicios, etc.

El riesgo de no tener una pronta y acertada respuesta aumenta cuando implica a una aeronave que se encuentra en vuelo y se le debe autorizar o denegar una actuación, especialmente si las decisiones están basadas solo en la habilidad para memorizar las normas que pueda tener el funcionario responsable. Adicionalmente, el problema se dificulta porque el RAC es una normativa que cambia continuamente debido a que debe atender la avalancha de avances tecnológicos y de procedimientos que se implementan año a año en el transporte aéreo, es por eso que, desde el año 2007 hasta el año 2019, el RAC ha tenido 25 versiones (Aerocivil, s.f.).

Si empleamos un sistema informático de búsqueda de términos convencional y no con inteligencia artificial, nos encontraremos con el mismo inconveniente de la pérdida de tiempo, debido a que el sistema no lograría interpretar nuestra pregunta particular y se limitaría a la búsqueda de un término específico, por lo que, solo nos mostraría en qué partes de los documentos se encuentra mencionado dicho término. Veamos un ejemplo sacado de la vida real: nos llega el aviso que, de las luces de borde de pista de un aeropuerto, se encuentra fuera de servicio uno de los dos circuitos, por lo que las luces están funcionando intercaladas (Una si y la otra no) ¿si es de noche, podremos seguir operando en este aeropuerto o se declara cerrado? Si se tuviera un sistema informático básico, se limitaría a la búsqueda de términos dentro de los reglamentos y si hacemos la búsqueda del término "luces de borde de pista" nos mostraría que de las 52 partes que componen el RAC, el término exacto se menciona 38 veces en 4 de sus partes, llamados RAC 4, RAC 14, RAC 24 y RAC 215. A pesar de acotar la búsqueda, un sistema informático convencional no sería capaz de indicarnos en dónde está la respuesta a nuestra duda y de todas formas se hace necesario hacer lectura a cada una de las 38 secciones hasta encontrar la respuesta. 
La inteligencia artificial permite agregar a los sistemas informáticos, características como: interpretación del lenguaje natural, Machine learning, reconocimiento visual, análisis de datos, análisis de texto, interacción contextual y un largo etcétera que permiten la realización de tareas parecidas a la inteligencia humana. Como lo dice Suarez (2018):

Principalmente la (I.A.) busca enfocarse en el análisis de como los seres humanos interpretan o buscan soluciones a la gran cantidad de problemas con los que se enfrentan diariamente, a través de la estrategia de búsqueda la cual tiene como pilar identificar la secuencia exacta de acciones que deben realizarse para encontrar su solución. En consecuencia, esta debe ser determinada mediante una exploración técnica de alternativas en cada uno de los posibles pasos a dar y en un proceso de "prueba y error", tratar de llegar al objetivo deseado, y así encontrar un camino o solución al problema planteado el cual haya generado un mayor porcentaje de confianza; este tipo de estrategia de resolución de problemas se conoce como "algoritmos de búsqueda" que es la implementación de sistemas informáticos que se encargan de buscar una información específica para generar la entrega de un respuesta concreta, un ejemplo claro es el buscador de Google.

El componente de Inteligencia Artificial permitiría no solamente una búsqueda de un término en particular en los textos de la legislación aeronáutica, sino que debería tener la capacidad de entender una consulta en lenguaje natural, interpretar la intención de la pregunta, realizar un análisis de texto en el RAC y dar como entregable una respuesta alineada con la normatividad. Estas poderosas herramientas informáticas permiten dar respuestas en segundos y la consecuente disminución de tiempo en la toma de decisiones.

\section{IDENTIFICACIÓN DE LOS INTERESADOS}

Se procedió a identificar y analizar a los interesados en el proyecto, basándose en el concepto "comunidad ATM" implementado por la Organización de Aviación Civil Internacional (OACI, 2005). Para entender la tabla, definimos los siguientes términos: 
- Poder: Nivel de autoridad en el proyecto.

- Interés: Nivel de preocupación en el proyecto.

- Influencia: Participación activa en el proyecto.

- Previsibilidad: Probabilidad de mantenerse alineado con el proyecto a pesar de los inconvenientes.

Tabla 1. Matriz de interesados

\begin{tabular}{|l|c|c|c|c|}
\hline \multicolumn{1}{|c|}{ Interesado } & Poder & Interés & Influencia & Previsibilidad \\
\hline Aerocivil como autoridad reguladora (AER) & Muy Alto & Muy Alto & Muy Alto & Muy alto \\
\hline $\begin{array}{l}\text { Explotadores de los aeródromos y sus } \\
\text { empleados (EXP) }\end{array}$ & Bajo & Muy Alto & Bajo & Alto \\
\hline $\begin{array}{l}\text { Empresas de transporte Aéreo y sus } \\
\text { empleados (EMP) }\end{array}$ & Alto & Muy Alto & Neutro & Alto \\
\hline Pasajeros (PAX) & Muy Bajo & Neutro & Muy Bajo & Bajo \\
\hline Aerocivil como proveedor de servicios (SNA) & Alto & Muy Alto & Alto & Alto \\
\hline Estado colombiano (EST) & Alto & Neutro & Neutro & Muy Alto \\
\hline Aduanas e inmigración (ADU) & Muy Bajo & Alto & Bajo & Alto \\
\hline Talleres de mantenimiento (TAL) & Bajo & Alto & Neutro & Alto \\
\hline Fabricantes de equipos (FAB) & Muy Bajo & Bajo & Muy Bajo & Alto \\
\hline Escuelas de formación aeronáutica (ESC) & Bajo & Muy Alto & Neutro & Alto \\
\hline Aeroclubes (ACB) & Bajo & Alto & Bajo & Alto \\
\hline Empresas de servicios especiales (ESP) & Bajo & Alto & Bajo & Alto \\
\hline
\end{tabular}

Fuente: elaboración propia

Matriz de poder - interés. Basándose en la Matriz de interesados, se realiza la Matriz de poder-interés para graficar el nivel de autoridad y preocupación respecto al proyecto: 


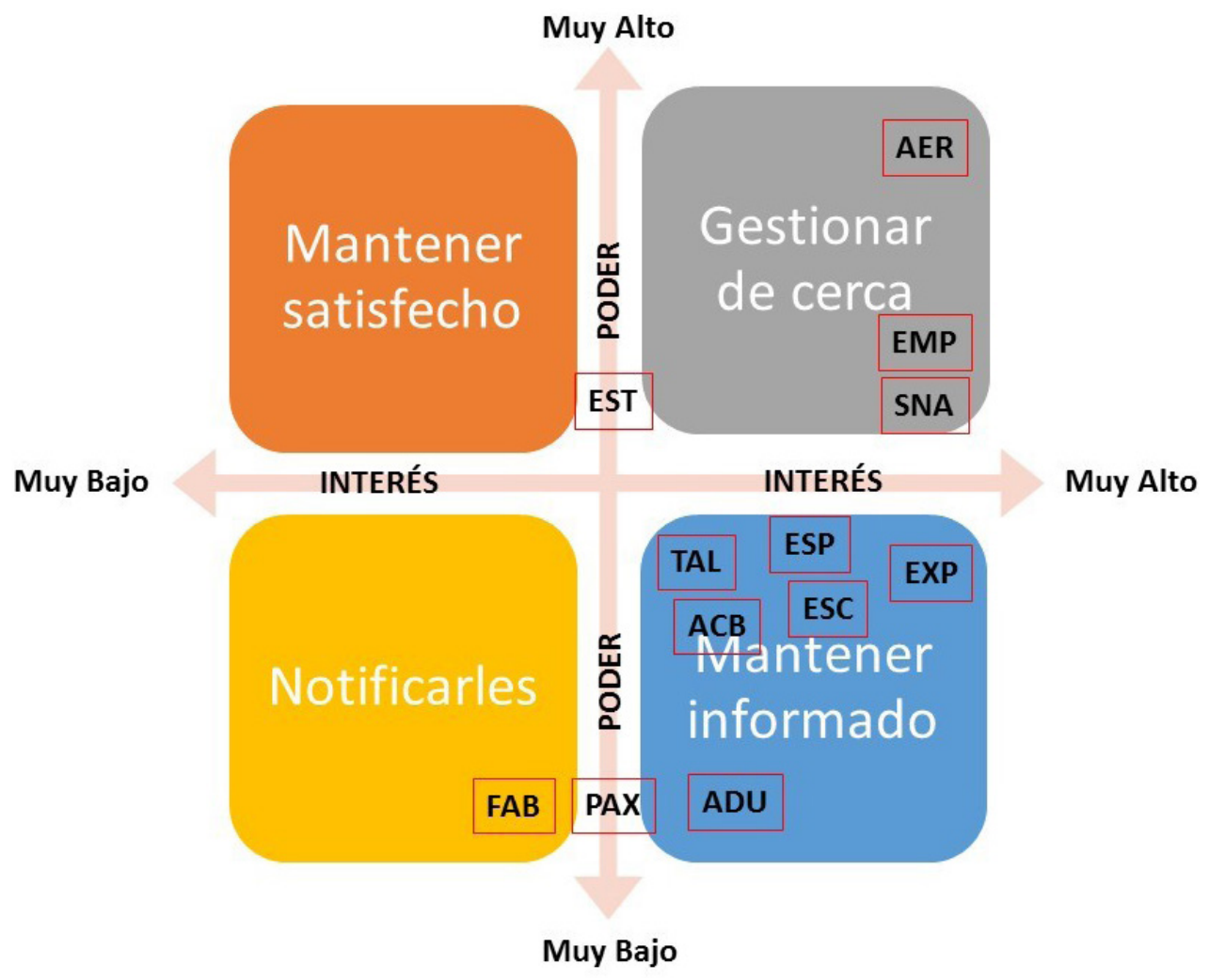

Figura 2. Matriz de poder - interés

Fuente: elaboración propia

La matriz nos muestra que, de los interesados, debemos trabajar muy de cerca con la Aerocivil por ser el ente regulador, las empresas explotadoras y la prestadora de los Servicios para la Navegación Aérea (Para Colombia es la misma Aerocivil). Para los demás interesados, será suficiente con mantenerlos informados.

Matriz influencia - previsibilidad. Basándose en la Matriz de interesados, se realiza la Matriz de influencia-previsibilidad para graficar el nivel de influencia y consistencia de los interesados respecto al proyecto: 


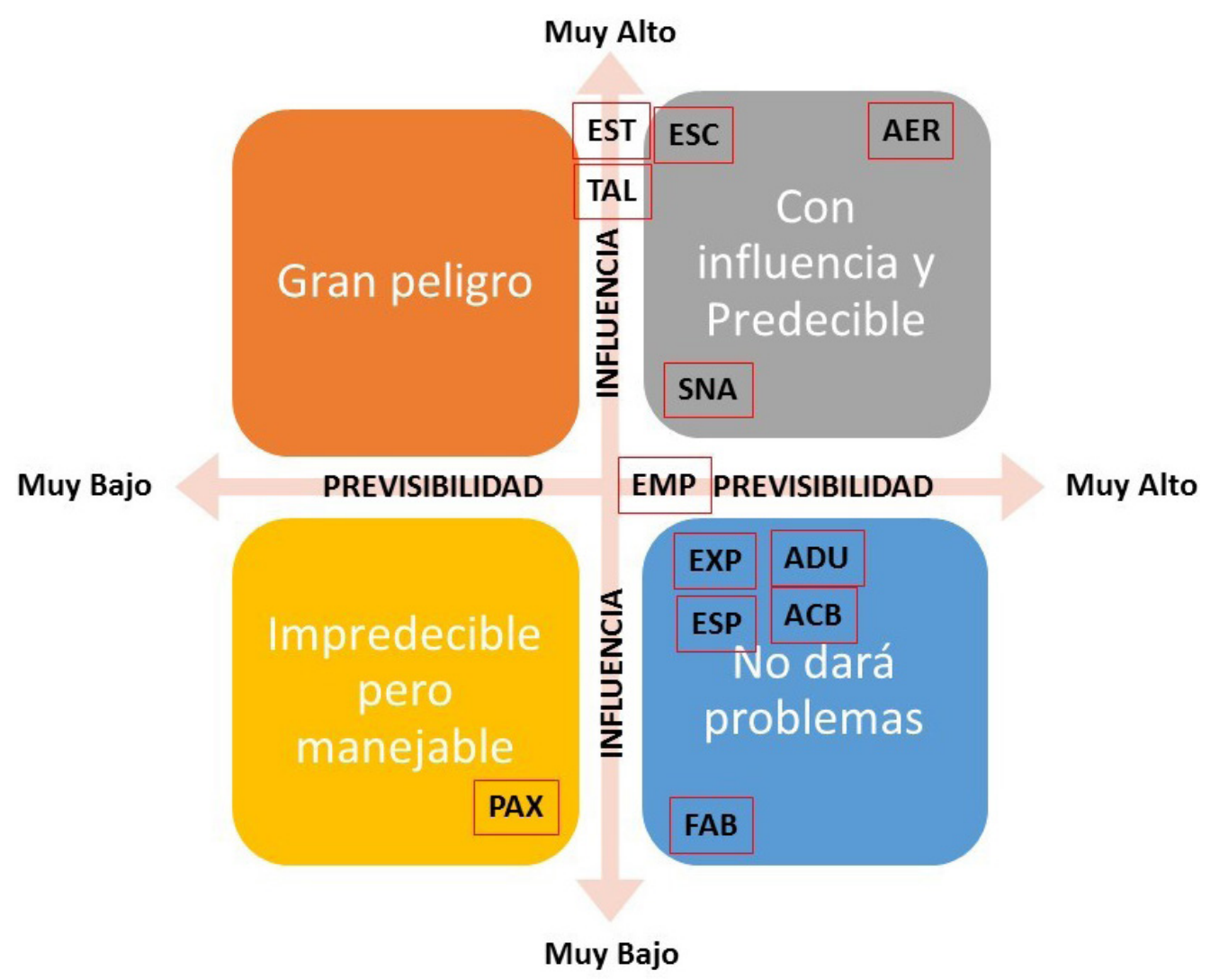

Figura 3. Matriz Influencia-Previsibilidad

Fuente: elaboración propia

La matriz nos muestra que, al trabajar con los interesados no habrá acciones imprevistas en las actividades de desarrollo del proyecto debido a hechos inesperados.

\section{IDENTIFICACIÓN DE LA MEJOR SOLUCIÓN EN TÉRMINOS DE IMPACTO Y EFECTIVIDAD}

Para poder realizar la correcta identificación de la mejor solución en términos de impacto y efectividad, se debe determinar las características mínimas que debe cumplir dicha solución.

Según Pérez y Cardoso (2015): “La búsqueda de respuestas (BR) con Inteligencia Artificial tiene como objetivo dar respuestas en lenguaje natural a preguntas también en lenguaje natural. Conceptualmente las aplicaciones de gestión de información no estructurada suelen organizarse en dos fases. En la fase de análisis se recogen y analizan colecciones de documentos 
y los resultados se almacenan en algún lenguaje o depósito intermedio. La fase de entrega hace accesible al usuario el resultado del análisis, y posiblemente el documento original completo mediante una interfaz apropiada.". La Figura 4 muestra la arquitectura básica de un sistema de búsqueda de respuestas.

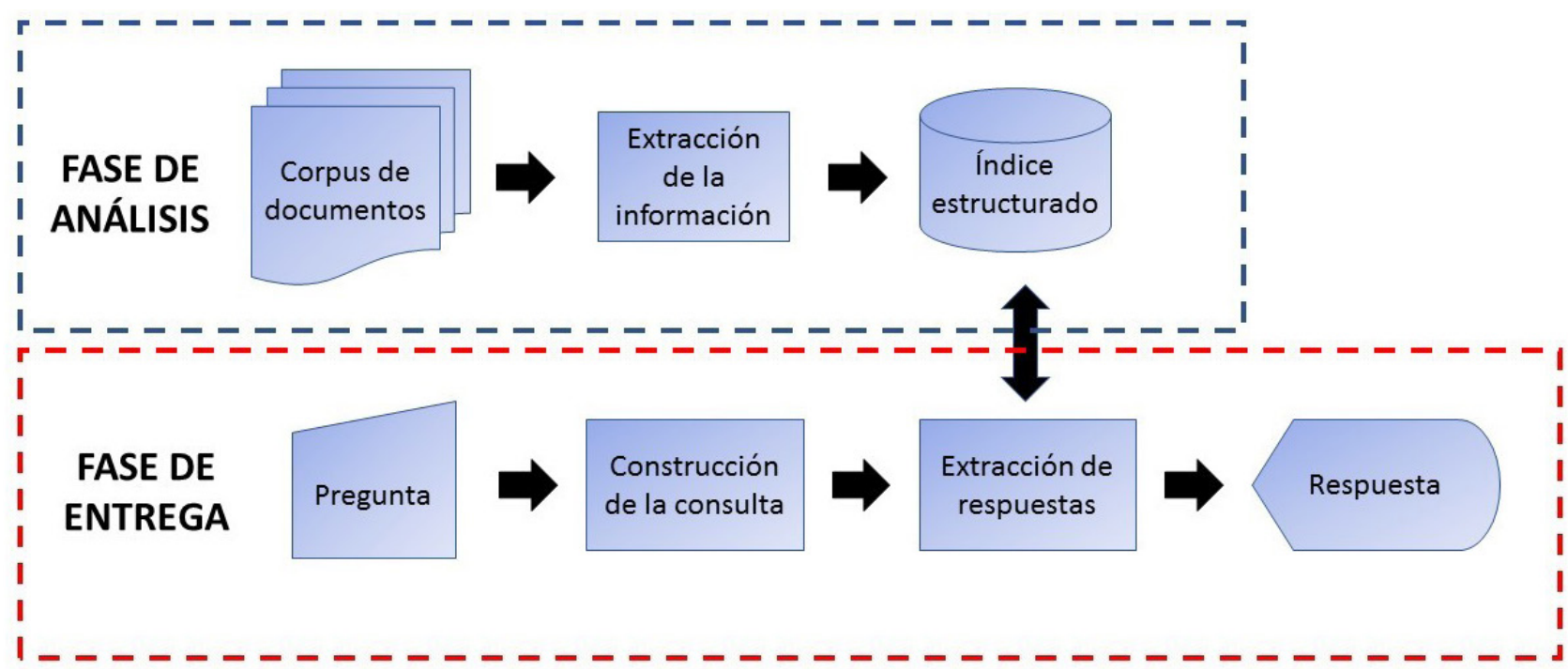

Figura 4. Arquitectura básica para la búsqueda de respuestas

Por lo anterior, las características mínimas buscadas son:

a. Extracción de información de un corpus de documentos con el fin de generar un índice estructurado.

b. Procesamiento de lenguaje natural y que lo realice en idioma español.

c. Análisis de preguntas y construcción de consultas.

d. Extracción y presentación de respuestas. 
Tomando esto en cuenta, se analizó a cada una de las posibles modalidades de solución que fueron encontradas en diversas fuentes documentales (Artículos de investigación, aplicaciones TIC y grandes plataformas de desarrollo), hallando a varias que contemplan las características deseadas, más sin embargo también se tuvo en cuenta costos, usabilidad, escalabilidad, soporte técnico, robustez y experiencia. Como consecuencia, se encontró que la mejor solución en términos de impacto y efectividad es la proporcionada por Google Cloud Al.

\section{RESTRICCIONES Y CONSTRICCIONES QUE DEBE ENFRENTAR LA SOLUCIÓN PROPUESTA}

Según el Project Management Institute (2017), los proyectos tienen por naturaleza, seis restricciones que pueden limitar su feliz término. De acuerdo a esta metodología, el presente proyecto tiene que tener en cuenta las siguientes restricciones:

Alcance. Se refiere al resultado esperado. Se requiere un sistema informático que gestione las dudas reglamentarias enmarcadas únicamente en el Reglamento Aeronáutico de Colombia (RAC) sin incluir las circulares reglamentarias u otras normativas que dependan del RAC.

Tiempo. Se refiere a los tiempos de culminación del proyecto. No existe una limitación a este respecto debido a que se encuentra en la etapa de propuesta, pero se puede planificar un tiempo de culminación de 9 meses en su desarrollo.

Coste. Se refiere al presupuesto disponible. La propuesta generará un coste de \$68’768.000, que será detallado más adelante.

Riesgo. Se refiere a qué puede salir mal y qué se puede hacer al respecto. Respecto al software de inteligencia artificial, el riesgo es que después de desarrollarlo, sus respuestas no sean apropiadas y puedan generar tomas de decisiones equivocadas, es decir, el mayor riesgo es que no sea fiable y esto se evitará en la meticulosidad del desarrollo del software.

Recursos. ¿Quién y qué se requiere para realizar el trabajo? Los recursos iniciales corresponden a un equipo de trabajo que desarrolle y entrene el software (Aproximadamente 6 personas) y equipo de cómputo que tenga acceso a las herramientas de desarrollo de Google. 
Calidad. ¿Qué tan cerca están los resultados y las expectativas? De acuerdo a los últimos avances en inteligencia artificial, es altamente probable desarrollar la herramienta informática propuesta con una alta fiabilidad en las respuestas dadas.

\section{PRESENTACIÓN DEL CASO DE NEGOCIO}

De acuerdo al Business Case Institute Scientific Management (Pereira \& Teixeira, 2015) un caso de negocio es "... un proceso de evaluación independiente y riguroso de una iniciativa que busca la creación de valor en la organización a través de la aplicación de medios financieros, humanos y de tiempo, en otras palabras, un caso de negocio consiste en una herramienta de toma de decisiones para determinar si una inversión crea valor". Con lo anterior en mente, procedemos a enumerar algunas consideraciones preliminares sobre el proyecto presentado.

\section{RECURSOS NECESARIOS PARA EL DESARROLLO DE LA SOLUCIÓN}

Tabla 2. Recursos necesarios para el desarrollo de la solución

\begin{tabular}{|c|c|c|c|}
\hline \multicolumn{4}{|c|}{ Recursos } \\
\hline Físicos & Intelectuales & Humanos & Económicos \\
\hline $\begin{array}{l}\text { - Tres (3) equipos de } \\
\text { cómputo de categoría } \\
\text { intermedia o superior. } \\
\text { - } \text { Acceso a internet } \\
\text { para conectarse a un } \\
\text { servidor especializado. } \\
\text { - Espacio de trabajo. }\end{array}$ & $\begin{array}{l}\text { Conocimientos en: } \\
\text { - Matemáticas (Álgebra } \\
\text { Lineal, Cálculo y } \\
\text { Estadística) } \\
\text { - Lenguaje de programación } \\
\text { (Python o cualquier otro } \\
\text { apropiado para Machine } \\
\text { Learning) } \\
\text { - Acceso y experticia en la } \\
\text { plataforma Google Cloud } \\
\text { Al. } \\
\text { - Derecho aeronáutico. }\end{array}$ & $\begin{array}{l}\text { - Un director de proyecto. } \\
\text { Mínimo cuatro } \\
\text { (4) expertos } \\
\text { desarrolladores de } \\
\text { Inteligencia Artificial. } \\
\text { - Un asesor en derecho } \\
\text { aeronáutico. }\end{array}$ & $\begin{array}{l}\text { - Costo de utilización de } \\
\text { la plataforma Google } \\
\text { Cloud Al. } \\
\text { - Costo de equipos de } \\
\text { cómputo. } \\
\text { - Costo de los recursos } \\
\text { humanos. } \\
\text { - Costo de } \\
\text { mantenimiento del } \\
\text { servidor. }\end{array}$ \\
\hline
\end{tabular}

Fuente: elaboración propia 


\section{RESULTADOS ESPERADOS E INDICADORES CLAVE DE DESEMPEÑO (KPI'S)}

Tabla 3. Resultados esperados e indicadores clave de desempeño (KPl`s)

\begin{tabular}{|l|l|}
\hline \multicolumn{1}{|c|}{ Resultados esperados } & \multicolumn{1}{|c|}{ Indicadores clave de desempeño (KPI's) } \\
\hline $\begin{array}{l}\text { Disminución en el tiempo de respuesta a las } \\
\text { consultas normativas. }\end{array}$ & Tiempo de respuesta promedio. \\
\hline Disminución de errores en la toma de decisiones. & $\begin{array}{l}\text { Número de decisiones erradas, basadas en } \\
\text { información incorrecta si usar la solución } \\
\text { informática. }\end{array}$ \\
\hline $\begin{array}{l}\text { Aumentar la seguridad operacional aeronáutica, } \\
\text { tomando decisiones correctas. }\end{array}$ & $\begin{array}{l}\text { Número de decisiones correctas, tomadas con } \\
\text { información de la solución informática. }\end{array}$ \\
\hline $\begin{array}{l}\text { Ser la mejor opción para ayudar a la toma de } \\
\text { decisiones en el ámbito aeronáutico. }\end{array}$ & $\begin{array}{l}\text { Indicadores clave de desempeño (KPI's) } \\
\text { - Cantidad de consultas por día. }\end{array}$ \\
\hline $\begin{array}{l}\text { Disminuir costos, por la no dilatación de toma de } \\
\text { decisiones. }\end{array}$ & $\begin{array}{l}\text { Cantidad de dinero ahorrado, al tomar decisiones } \\
\text { con el sistema informático, respecto a: } \\
\text { - Consumo de combustible. }\end{array}$ \\
\hline $\begin{array}{l}\text { Ayudar a la redacción del RAC y evitar } \\
\text { incongruencias. }\end{array}$ & $\begin{array}{l}\text { Retrasos. } \\
\text { - Gastos legales. }\end{array}$ \\
\hline
\end{tabular}

Fuente: elaboración propia

\section{SOLUCIÓN AL PROBLEMA TÉCNICO}

\section{ANÁLISIS DE ESCENARIOS}

El análisis prospectivo se basa en un sinnúmero de herramientas que le permiten al tomador de decisiones sopesar las opciones de las que dispone y las posibles consecuencias. La base del análisis prospectivo se encuentra en la herramienta llamada análisis de escenarios y en donde, de acuerdo a Godet (2000), un escenario es "un conjunto formado por la descripción de una situación futura y de la trayectoria de eventos que permiten pasar de una situación origen a una situación futura". 
Basándonos en los factores clave encontrados y las proyecciones futuras, procedimos a ponerlos en interacción para construir los posibles escenarios a analizar y conforme al cruce de las proyecciones futuras se construyeron los posibles escenarios a los que nos deberíamos enfrentar y que serán la base para plantear las estrategias que nos ayuden a mitigar los imprevistos y permitan salvar los obstáculos que se puedan presentar.

Tomamos el escenario que condensaba el mayor número de posibles obstáculos y así abarcar todas las posibles estrategias.

Tabla 4. Análisis de escenarios - Estrategias

\begin{tabular}{|l|l|}
\hline \multicolumn{1}{|c|}{ Escenario } & \multicolumn{1}{c|}{ Posibles estrategias } \\
\hline $\begin{array}{l}\text { Que el nivel directivo de la } \\
\text { Aerocivil niegue la iniciativa. }\end{array}$ & $\begin{array}{l}\text { 1. Mostrar los beneficios de su implementación. } \\
\text { 2. Establecer medidores de seguimiento que refuercen los beneficios. } \\
\text { 3. Enfatizar los elevados costos de la no implementación }\end{array}$ \\
\hline $\begin{array}{l}\text { Que el sistema cometa } \\
\text { demasiados errores al encontrar } \\
\text { respuestas adecuadas. }\end{array}$ & $\begin{array}{l}\text { 1. Mejorar los algoritmos de búsqueda de respuestas. } \\
\text { 2. Mejorar los algoritmos de entrenamiento del sistema. }\end{array}$ \\
\hline $\begin{array}{l}\text { Que los usuarios no utilicen } \\
\text { el sistema. }\end{array}$ & $\begin{array}{l}\text { 1. Establecer una campaña de marketing que le de visibilidad. } \\
\text { 2. Promulgar eventos de capacitación sobre el uso del sistema. }\end{array}$ \\
\hline
\end{tabular}

Fuente: elaboración propia

Como se puede evidenciar, existen una gran variedad de estrategias que permiten solventar la mayor cantidad de obstáculos y que permitirán que el proyecto llegue a feliz término.

\section{ANÁLISIS DE CAUSAS Y EFECTOS}

Para el análisis de causa y efectos, nos basaremos en el diagrama de Ishikawa, que es utilizado para explorar todas las causas reales o potenciales (entradas) que explican un efecto de interés (salida) (Ishikawa, 1990). 


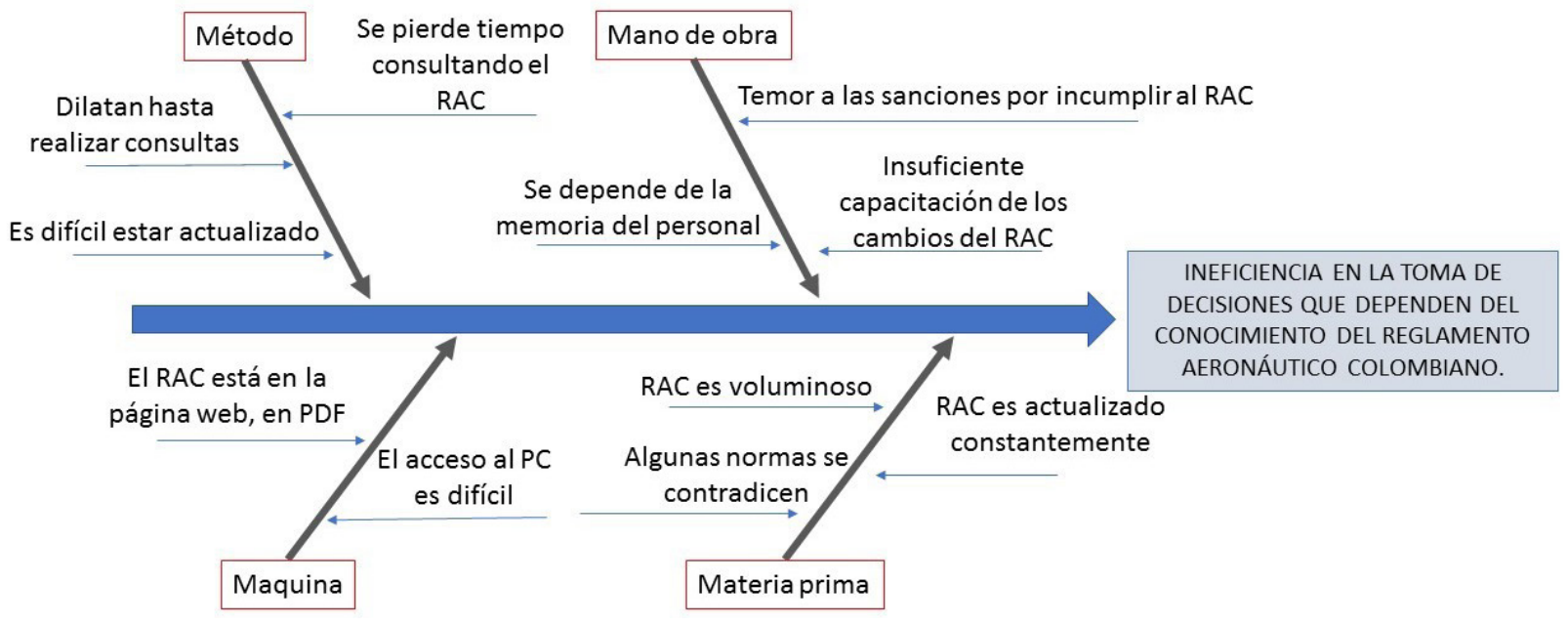

Figura 5. Gráfica de causa y efecto

Fuente: elaboración propia

\section{ALINEACIÓN DE LA SOLUCIÓN CON LOS OBJETIVOS ESTRATÉGICOS DE AEROCIVIL}

Para conocer los objetivos estratégicos de la Aerocivil, nos debemos remitir al Plan Estratégico Aeronáutico (PEA 2030) (Aerocivil, 2019a), que está articulado con el Plan Nacional de Desarrollo (PND) 2018-2022 "Pacto por Colombia, pacto por la equidad".

La visión contemplada en este plan es: "Movilizar 100 millones de pasajeros y duplicar el transporte de carga en un entorno institucional claro, competitivo, conectado, seguro y sostenible, soportado en una infraestructura renovada, una industria robustecida y un talento humano de excelencia.".

Su desarrollo se consolida en la ejecución de los objetivos trazados en siete (7) ejes temáticos que lo sustentan: institucionalidad, Conectividad, Competitividad, Infraestructura y sostenibilidad ambiental, Industria Aeronáutica y cadena de suministro, Seguridad operacional y Seguridad de la aviación civil y Desarrollo del talento Humano del sector.

De todos los objetivos específicos, podemos enumerar los que se alinean con la solución propuesta en el presente trabajo: 
Tabla 5. Objetivos estratégicos alineados con la solución propuesta

\begin{tabular}{|l|l|}
\hline \multicolumn{1}{|c|}{ Objetivo estratégico } & \multicolumn{1}{c|}{ Objetivos específicos } \\
\hline Competitividad & $\begin{array}{l}\text { Contar con mecanismos efectivos para la protección de los derechos de los } \\
\text { usuarios, frente a los servicios ofrecidos en el transporte aéreo, para mejorar } \\
\text { la calidad y satisfacción de éstos. }\end{array}$ \\
\hline $\begin{array}{l}\text { Seguridad operacional y de la } \\
\text { aviación civil }\end{array}$ & $\begin{array}{l}\text { Apropiar en el sector las mejores prácticas en seguridad operacional de la } \\
\text { OACl, con el fin de corregir la brecha en la implementación de los elementos } \\
\text { críticos del sistema de vigilancia de la seguridad operacional. } \\
\text { - Mejorar la capacidad del Estado en la aplicación de un sistema de Vigilancia } \\
\text { de la Seguridad Operacional basado en riesgos, disponiendo de mecanismos } \\
\text { para la compilación, transformación y administración de datos de seguridad } \\
\text { operacional (Safety BIG DATA), que permitan generar análisis estadísticos para } \\
\text { la toma de decisiones. }\end{array}$ \\
\hline Desarrollo del talento humano \\
en el sector & $\begin{array}{l}\text { Disponer de un sistema de gestión del Talento Humano que permita responder } \\
\text { a las necesidades del Sector Aeronáutico en cantidad y calidad. } \\
\text { Alcanzar una amplia oferta de capacitación orientada a la gestión aeronáutica } \\
\text { integral, en todos los niveles y campos. }\end{array}$ \\
\hline
\end{tabular}

Fuente: elaboración propia

\section{PLAN DE COMUNICACIONES}

Según el Project Management Institute (2017), el plan de gestión de las comunicaciones es un componente del plan para la dirección del proyecto que describe la forma en que se planificarán, estructurarán, implementarán y monitorearán las comunicaciones del proyecto para lograr la eficacia.

El plan contendrá los requisitos de comunicación de los stakeholders, dependiendo de los intereses y grado de influencia sobre el éxito del proyecto, tomando en cuenta que, a mayor grado de intereses e influencia en el proyecto, las comunicaciones deberán ser más frecuentes y cercanas. Adicionalmente, se estableció que la información se comunicará en idioma español y se utilizarán diferentes métodos y metodologías (Que se mostrarán en la figura 6).

La persona responsable de la comunicación será el director del proyecto, la cual utilizará los siguientes métodos, dependiendo si el requisito de comunicaciones es cercano o no: 


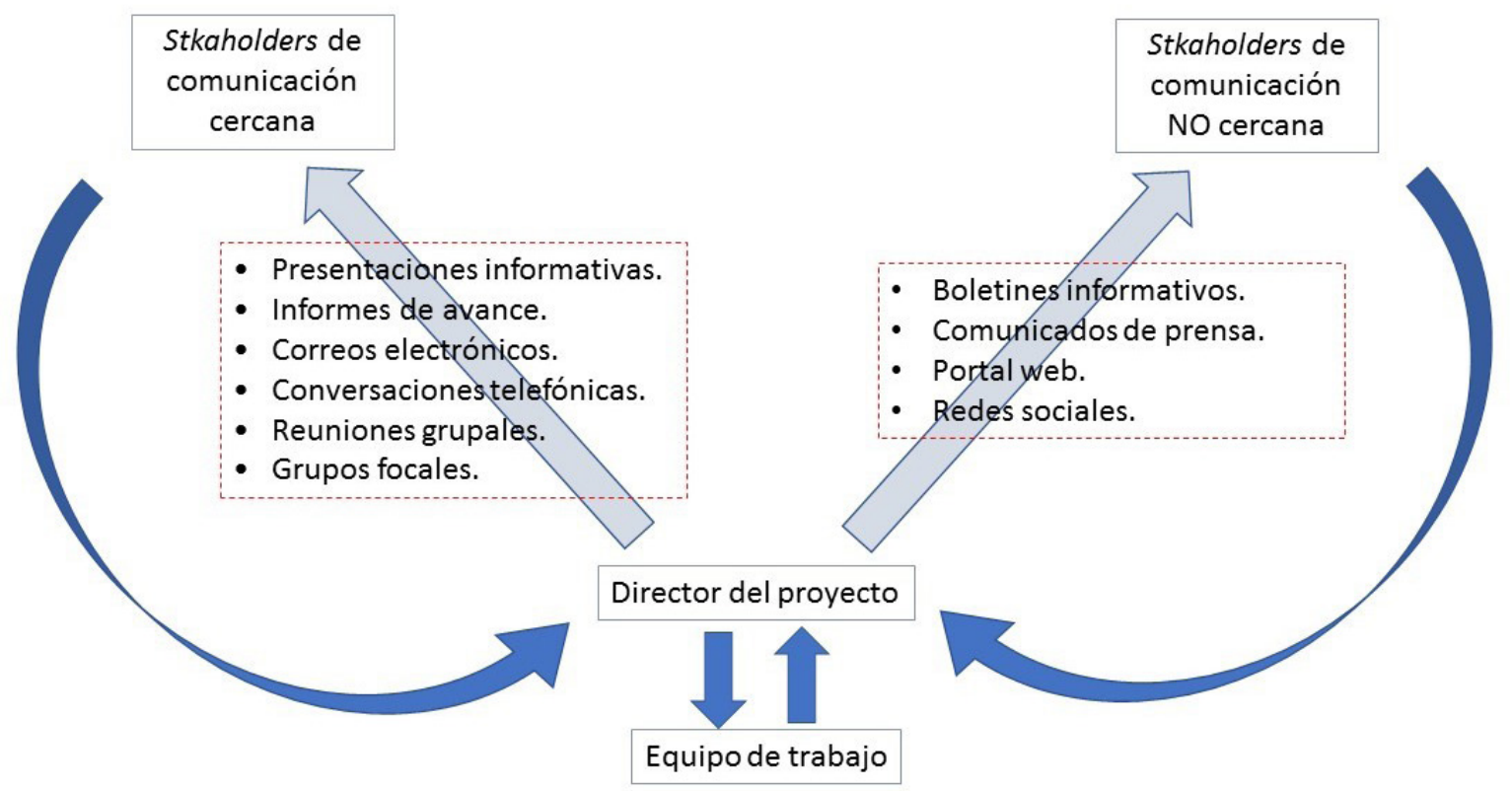

Figura 6. Flujo de información del proyecto

Fuente: elaboración propia

ASPECTOS ADMINISTRATIVOS

Para los aspectos administrativos, se utilizó la herramienta Microsoft Project, a la cual se incorporaron todos los datos relevantes del proyecto.

Se mostrará el cronograma de actividades resumido, los costos en que se incurrirán, lo entregables y la factibilidad económica del proyecto. 


\section{CRONOGRAMA DE ACTIVIDADES}

\begin{tabular}{|c|c|c|c|c|c|c|c|c|c|}
\hline Nombre de la actividad & MES 1 & MES 2 & MES 3 & MES 4 & MES 5 & MES 6 & MES 7 & MES 8 & MES 9 \\
\hline \multicolumn{10}{|l|}{ Equipos y software } \\
\hline \multicolumn{10}{|c|}{ Compra e instalación de equipos de computo } \\
\hline \multicolumn{10}{|l|}{ Programación del software } \\
\hline \multicolumn{10}{|c|}{ Desarrollo de la fase de análisis } \\
\hline \multicolumn{10}{|c|}{ Desarrollo de la fase de entrega } \\
\hline \multicolumn{10}{|c|}{ Pruebas del sistema } \\
\hline \multicolumn{10}{|c|}{ Instalación en página web/servidor } \\
\hline \multicolumn{10}{|c|}{ Plan de comunicaciones } \\
\hline \multicolumn{10}{|c|}{ Informes de avances del proyecto } \\
\hline \multicolumn{10}{|c|}{ Informe de puesta en marcha del sistema } \\
\hline \multicolumn{10}{|l|}{ Presentación del proyecto } \\
\hline Capacitación de uso & & & & & & & & & \\
\hline
\end{tabular}

Figura 7. Cronograma de actividades

Fuente: elaboración propia

\section{COSTOS DEL PROYECTO DE DESARROLLO TECNOLÓGICO}

\section{ESTADO DEL COSTO \\ Estado de costo para las tareas de nivel superior.}

$\$ 60.000 .000,00$

$\$ 50.000 .000,00$

$\$ 40.000 .000,00$

$\$ 30.000 .000,00$

$\$ 20.000 .000,00$

$\$ 10.000 .000,00$

$\$ 0,00$

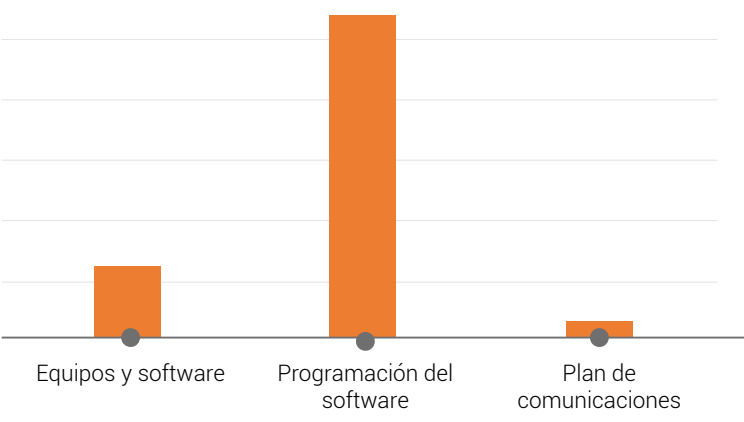

Costo real

Costo restante

- Costo línea base

DETALLES DE COSTOS

Detalles de costos para todas las tareas

de nivel superior

\begin{tabular}{|l|c|}
\hline \multicolumn{1}{|c|}{ Nombre } & Costo \\
\hline Equipo y software & $\$ 12.288 .000,00$ \\
\hline Programación del software & $\$ 53.928 .000,00$ \\
\hline Plan de comunicaciones & $\$ 1.2560 .000,00$ \\
\hline
\end{tabular}

Figura 8. Costos del proyecto

Fuente: elaboración propia

\section{El costo total del proyecto sería de $\$ 68^{\prime} 768.000$}




\section{RELACIÓN DE ENTREGABLES DEL PROYECTO DE CONSULTORÍA}

De acuerdo al Project Management Institute (2017), los proyectos se llevan a cabo para cumplir objetivos mediante la producción de entregables, un entregable lo definen como cualquier producto, resultado o capacidad único y verificable para ejecutar un servicio que se produce para completar un proceso, una fase o un proyecto. Los entregables pueden ser tangibles o intangibles.

Para el presente proyecto, los entregables serían:

- Estudio de viabilidad.

- Software de inteligencia artificial para el manejo de dudas normativas basadas en el RAC.

- Documentación del software:

- Informe de las pruebas.

- Informe final

EVALUACIÓN DEL DESARROLLO DEL PROYECTO EN TÉRMINOS DE FACTIBILIDAD ECONÓMICA

El valor del negocio en los proyectos se refiere al beneficio que los resultados de un proyecto específico proporcionan a sus interesados. El beneficio de los proyectos puede ser tangible, intangible o ambos.

Tomando como base la información sobre el presupuesto 2020 de la Aerocivil detallado en el Decreto 2411 (Ministerio de Hacienda y Crédito público, 2019), que es un total de \$271 billones de pesos y dentro del cual, existe un rublo llamado "Fortalecimiento de la gestión interna para la alineación de la estrategia TI con los componentes misionales, para crear una competitividad estratégica en la Unidad Administrativa Especial De Aeronáutica Civil a nivel nacional" con una asignación presupuestal de veinticinco mil millones de pesos (\$25.000'000.000), nos lleva a concluir que el costo del proyecto no representaría una cifra importante dentro de su presupuesto. 
Si, además, observamos los principales beneficios de la puesta en marcha del sistema que son:

- Disminución de costos por demoras (Para las aerolíneas, para los pasajeros y para el medio ambiente teniendo en cuenta el ahorro de combustible)

- Disminución de costos de defensa legal.

Nos lleva a evaluar que el desarrollo del proyecto no solo el factible económicamente hablando, sigo que llevará a grandes disminuciones de costos a la Aerocivil y a sus stakeholders y acercará a la institución a la consecución de sus objetivos estratégicos.

\section{CONCLUSIONES}

Actualmente, la toma de decisiones de los diferentes actores de la aviación depende en gran medida de su capacitación, por lo que en última instancia se está dependiendo de la capacidad de memorizar los reglamentos, y en caso de duda, se debe remitir a la fuente para poder tomar la decisión. El formato en que actualmente se encuentra el RAC (Archivos PDF en la página web de la Aerocivil) hace que sea dispendioso el aclarar alguna duda, por lo que afecta la eficiencia del sistema.

Al tratar de resolver el problema técnico, se descubre que ya existen las herramientas tecnológicas necesarias para implementar un sistema informático basado en inteligencia artificial que pueda interpretar el lenguaje natural hablado o escrito, que descifre las intenciones del interlocutor y apoyado en una base de datos relacional, dar una respuesta en tiempo real a cualquier duda que se tenga respecto al corpus de documentos que enmarcan al Reglamento Aeronáutico de Colombia.

De forma específica, se logró determinar que es posible la implementación de un sistema informático con inteligencia artificial para resolver consultas normativas en el ámbito de los Reglamentos Aeronáuticos de Colombia (RAC), el cual permitiría la disminución de los tiempos de respuesta a las consultas normativas, se disminuiría la cantidad de errores en la toma de decisiones y se podría aumentar la seguridad operacional aeronáutica; esto redundará en la disminución de los costos operacionales, ambientales y legales. 
Con los resultados del proyecto, a futuro se podrá generalizar su aplicación en otros ámbitos, podría facilitar la toma de decisiones en cualquier área funcional de diferentes organizaciones, democratizar el acceso a la defensa de los intereses sin necesidad de contar con un profesional del derecho, permitiría la reducción de tiempos de respuesta a cualquier actuación (Derechos de petición), asistiría a los empresarios y profesionales de todas las áreas en su toma de decisiones cotidianas donde se necesite la aplicación de sistemas para la solución rápida de consultas. Por ejemplo, imaginemos a un empresario que debe asegurarse que todas sus decisiones y actuaciones estén enmarcadas en lo dictado por la ley, pero no cuenta con los recursos económicos suficientes para contar de tiempo completo con un abogado experto que lo asesore en los pormenores. Este empresario podría utilizar un software con inteligencia artificial que le resuelva dudas en áreas como la gerencia, talento humano, finanzas, producción, legal, marketing, servicio al cliente, etc. (Sobre qué impuestos debe pagar y cuándo, cuáles con los requisitos que debe cumplir si decide sacar un nuevo producto al mercado, cuáles debería ser las condiciones de etiquetado, empaque y registro ante entes gubernamentales, ¿es necesario una patente?, si decide ampliar la planta de producción necesita cumplir una nueva reglamentación?, etc.)

"Es por ello que toda persona debe saber en lo general, dentro del ámbito de las actividades que realiza, el marco jurídico que lo regula. También debe entender el alcance legal de las leyes que se le pueden aplicar." (Hooper, 2015)

\section{REFERENCIAS}

Aerocivil. (s.f.). Históricos RAC. http://www.aerocivil.gov.co/normatividad/Pages/Historicos-RAC.aspx

Aerocivil. (2019a) Plan Estratégico Aeronáutico (PEA 2030) - Resumen ejecutivo. http://www. aerocivil.gov.co/cea/QuienesSomos/Catedra\%202030/Resumen\%20Ejecutivo\%20Plan\%20 Estrate\%CC\%81gico\%20Aerona\%CC\%81utico\%202030.pdf

Aerocivil. (2019b). Reglamentos Aeronáuticos de Colombia - Parte 1. http://www.aerocivil.gov.co/normatividad/RAC/RAC\%20\%201\%20-\%20Definiciones.pdf

Business Case Institute. (2019). What is a Scientific Business Case. http://www.businesscaseinstitute. org/bcbok-standards/what-is-a-business-case 
Código de Comercio [CC]. Decreto 140 de 1971. Junio 16 de 1971 (Colombia)

Congreso de Colombia. (16 de mayo de 2017). Por el cual se modifica la estructura de la Unidad Administrativa Especial de Aeronáutica Civil (Aerocivil), y se dictan otras disposiciones. [Decreto 823 de 2017]. DO: 50.235

Fabra, J. L., y Núñez, A. (2015). Enciclopedia de Filosofía y Teoría del Derecho (Vol. 1). México: UNAM, Instituto de Investigaciones Jurídicas.

Fernández, C., y Boulat, P. (2015). Inteligencia Artificial y Derecho. Problemas y perspectivas. http:// noticias.juridicas.com/conocimiento/articulos-doctrinales/9441-inteligencia-artificial-yderecho-problemas-y-perspectivas/

Godet, M., Monti, R., Meunier, F., y Roubelat, F. (2000). La caja de herramientas de la prospectiva estratégica. https://hal-lara.archives-ouvertes.fr/hal-02185401/document

Hernández, N. B., Navarrete Luque, C. E., León Segura, C. M., Real López, M. de J., Chiriboga Hungría, J. A., y Ricardo, J. E. (2019). La toma de decisiones en la informática jurídica basado en el uso de los sistemas expertos. Investigación Operacional, 40(1), 131-139.

Hooper, P. (2015, diciembre 24). ¿No conocer la ley me libera de su cumplimiento? [Web log post]. https:// www.entrepreneur.com/article/269402

Ishikawa, K. (1990). Introduction to quality control. Productivity Press.

Méndez, C. (2006). Metodología, diseño y desarrollo del proceso de investigación con énfasis en ciencias empresariales. Editorial Limusa, cuarta edición, Colombia.

Ministerio de Hacienda y Crédito público. (30 de diciembre de 2019). Por el cual se liquida el Presupuesto General De La Nación para la vigencia fiscal de 2020. [Decreto 2411 del 30 de diciembre de 2019] 
Narváez, C. (2018, septiembre 14). Inteligencia artificial para el sistema judicial colombiano: opinión [Web log post]. (https://www.ambitojuridico.com/noticias/tecnologia/tic/inteligencia-artificialpara-el-sistema-judicial-colombiano-opinion)

Organización de Aviación Civil Internacional (OACI). (2005). Doc 9854 - Concepto operacional de gestión del tránsito aéreo mundial. Primera edición.

Pereira, Leandro; Teixeira, Claudia. (2016). A guide to the business case body of knowledge. Business case institute scientific management. http://www.businesscaseinstitute.org/bcbok-standards/ bcbok-guide

Pérez Abelleira, M. A., Cardoso, A. C., y Bini, A. (2015, May). Minería de textos: sistemas de búsqueda de respuestas. In XVII Workshop de Investigadores en Ciencias de la Computación (Salta, 2015).

Project Management Institute. (2017). La guía de los fundamentos para la dirección de proyectos (Guía del PMBOK). Project Management Institute, Inc.

Rodríguez, P. (2020). Solución informática con inteligencia artificial para resolver consultas normativas en el ámbito de los Reglamentos Aeronáuticos de Colombia (RAC). https://repository.unad.edu.co/ handle/10596/35166.

Suarez Prieto, J. E. (2018). Los beneficios de la inteligencia artificial en el sector empresarial. Tesis de grado. Universidad Santo Tomás. Bogotá. Recuperado de https://repository.usta.edu.co/bitstream/ handle/11634/12948/2018juliosuarez.pdf?sequence=1\&isAllowed=y

Texo Denes, A. (2017). Ignorance and opacity: a view to criminal law. Revista De La Facultad De Derecho, (42), 281-314.

UNESCO (s.f.). Árbol de problemas. http://www.unesco.org/new/es/culture/themes/\%20culturaldiversity/diversity-of-cultural\%20expressions/tools/policy-guide/planificar/diagnosticar/ arbol-de-problemas/ 